\title{
Preparation of harvested skin using the Versajet Hydrosurgery System in full-thickness skin grafts
}

\author{
Seo Gil Choi, Hyun Woo Shin, Kun Chul Yoon \\ Department of Plastic and Reconstructive Surgery, Kangbuk Samsung Hospital, Sungkyunkwan University School of Medicine, Seoul, Korea
}

During a full-thickness skin graft procedure, the thickness of the harvested donor skin is adjusted based on the location of the recipient site and the judgment of the surgeon. Conventionally, the thickness of the harvested skin is roughly adjusted using surgical instruments such as scalpels and scissors. However, this method is not only time-consuming, but also requires effort to obtain both the desired thickness of the harvested skin and a smooth surface of that skin. Moreover, there is a possibility of skin perforation. Hence, the authors devised a method of adjusting harvested skin thickness using the Versajet Hydrosurgery System. The Versajet device is a handheld hydrosurgical tool that delivers a high-speed jet stream of saline solution, which enables the precise debridement of tissue. This method makes it easier and faster for the surgeon to obtain the desired thickness of harvested skin. In addition, by obtaining a smooth surface and an even thickness of harvested donor skin, this technique may lead to improved graft viability.

Keywords Skin transplantation / Wounds and Injuries / Wound healing
Correspondence: Hyun Woo Shin Department of Plastic and Reconstructive Surgery, Kangbuk Samsung Hospital, Sungkyunkwan University School of Medicine, 29 Saemunan-ro, Jongno-gu, Seoul 03181, Korea

Tel: +82-2-2001-2178

Fax: +82-2-2001-2177

E-mail:mdshin7@naver.com

This article was presented as a poster at the 9th Research and Reconstruction Forum on May 9-10, 2019, in Gwangju, Korea.

Received: June 15, $2019 \bullet$ Revised: August 14, $2019 \bullet$ Accepted: September 19, 2019

pISSN: 2234-6163 • elSSN: 2234-6171 • https://doi.org/10.5999/aps.2019.00745 • Arch Plast Surg 2019;46:603-607

\section{INTRODUCTION}

Skin grafting, including split-thickness skin graft (STSG) and full-thickness skin graft (FTSG) surgery, has been regarded as a relatively simple procedure to cover skin defects. However, poor skin quality and frequent scar contracture are well-known problems associated with STSG. Moreover, in functional regions that experience strain and have high requirements of elasticity, pliability, and stability, such as joint areas, it is very important to reconstruct the defect as similarly as possible to the original tissue to preserve the full range of motion. FTSG is durable, exhibits minimal contraction, and typically produces good cosmetic results. In comparison to STSG, FTSG is also associated with reduced donor site morbidity because primary closure leads to faster healing and less pain [1].
The thickness of the donor skin is a very important consideration of the FTSG procedure because it affects postoperative viability and contracture. Conventionally, the thickness of the harvested skin is regulated using a manual method involving surgical instruments such as scalpels, scissors, and Metzenbaum scissors. This manual procedure is time-consuming and makes it difficult to precisely attain the thickness judged optimal by the surgeon. Moreover, the manual technique tends to result in an irregular surface of the harvested skin.

The Versajet Hydrosurgery System (Smith \& Nephew, Memphis, TN, USA) is a device designed for surgical debridement based on the Venturi effect, wherein a high-pressure water jet is pushed through a suitable hose to the tip of a handpiece while simultaneously cutting and aspirating debris. In general, this device is used for tangential hydrodebridement [2]. The authors 
of this study used this device for recipient site debridement before skin grafting. As described herein, we also attempted to use the Versajet to modify the thickness of the harvested skin.

The study was approved by the Institutional Review Board of Kangbuk Samsung Hospital (IRB No. KBSMC 2019-07-052) and was performed in accordance with the principles of the Declaration of Helsinki. Informed consent was obtained from all patients.

\section{IDEAS}

\section{Idea 1}

A 12-year-old girl was referred to our plastic surgery department due to a soft tissue defect of the cubital fossa caused by cellulitis. The size of the defect was $6 \mathrm{~cm} \times 4 \mathrm{~cm}$ with a well-vascularized

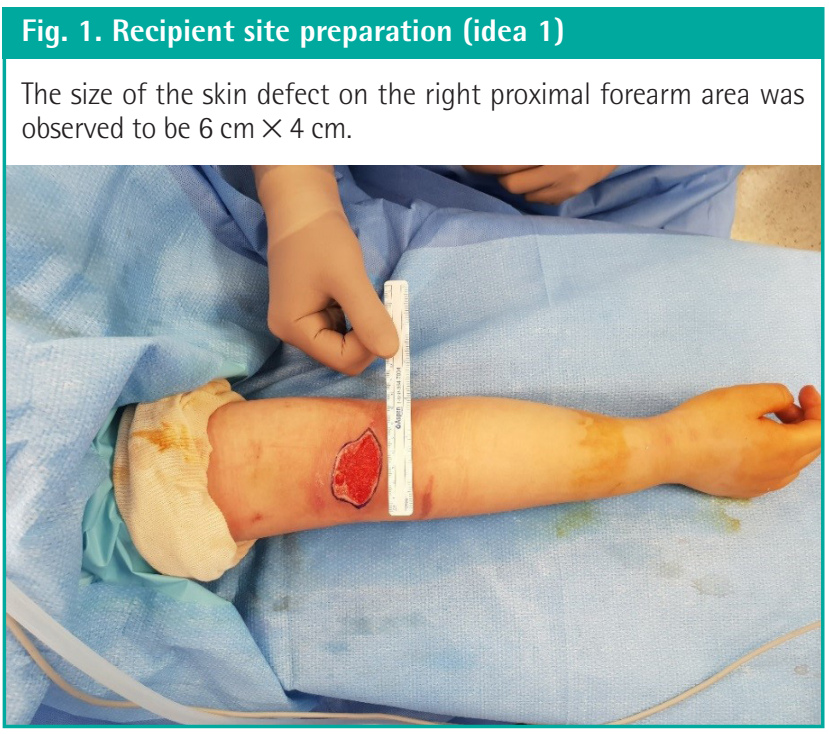

granulation bed. Patient compliance was poor due to an intellectual disability. Hence, we opted for FTSG instead of flap surgery due to the expectation of poor patient cooperation after surgery. As shown in Fig. 1, the donor skin was harvested from the right inguinal area. The elliptical incision line was designed to be slightly enlarged to allow for the contraction of the recipient site after grafting. The skin was elevated at the depth of the subcutaneous tissue using a scalpel and iris scissors. The harvested skin was stretched and fixed on overlapping surgical towels. Subsequently, the undersurface of the harvested skin was defatted and trimmed using the Versajet device until the desired thickness was obtained (Fig. 2). The angle of the Versajet handpiece was $45^{\circ}$. The initial power setting for this procedure was 3 , and it was later lowered to 2 for more precise control. Using this procedure, the precise desired thickness of the harvested skin

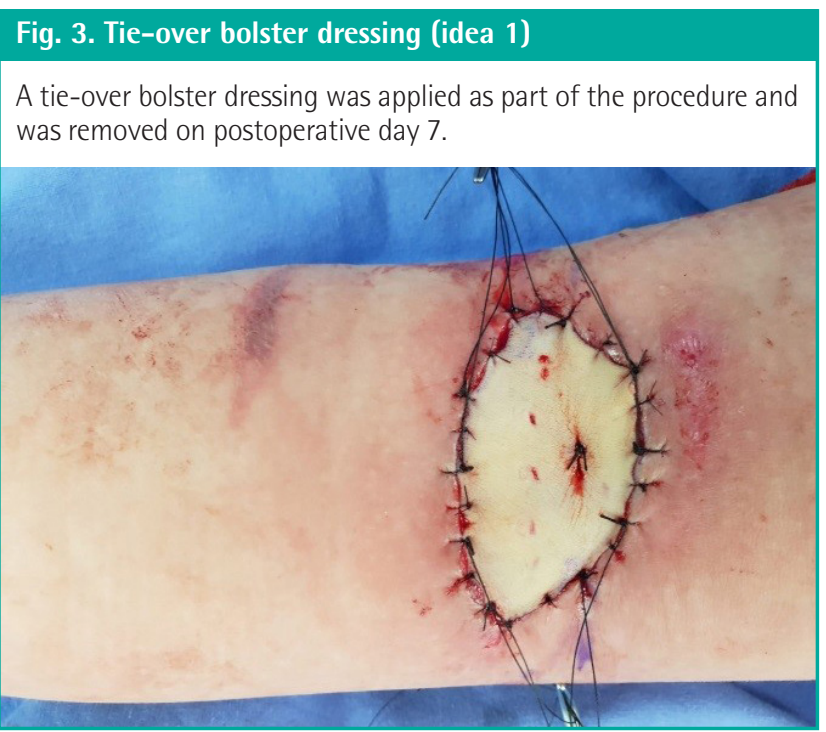

Fig. 2. Preparation of harvested donor skin (idea 1)

(A) The harvested skin was defatted and trimmed using the Versajet Hydrosurgery System. (B) The desired thickness of the harvested skin was obtained.
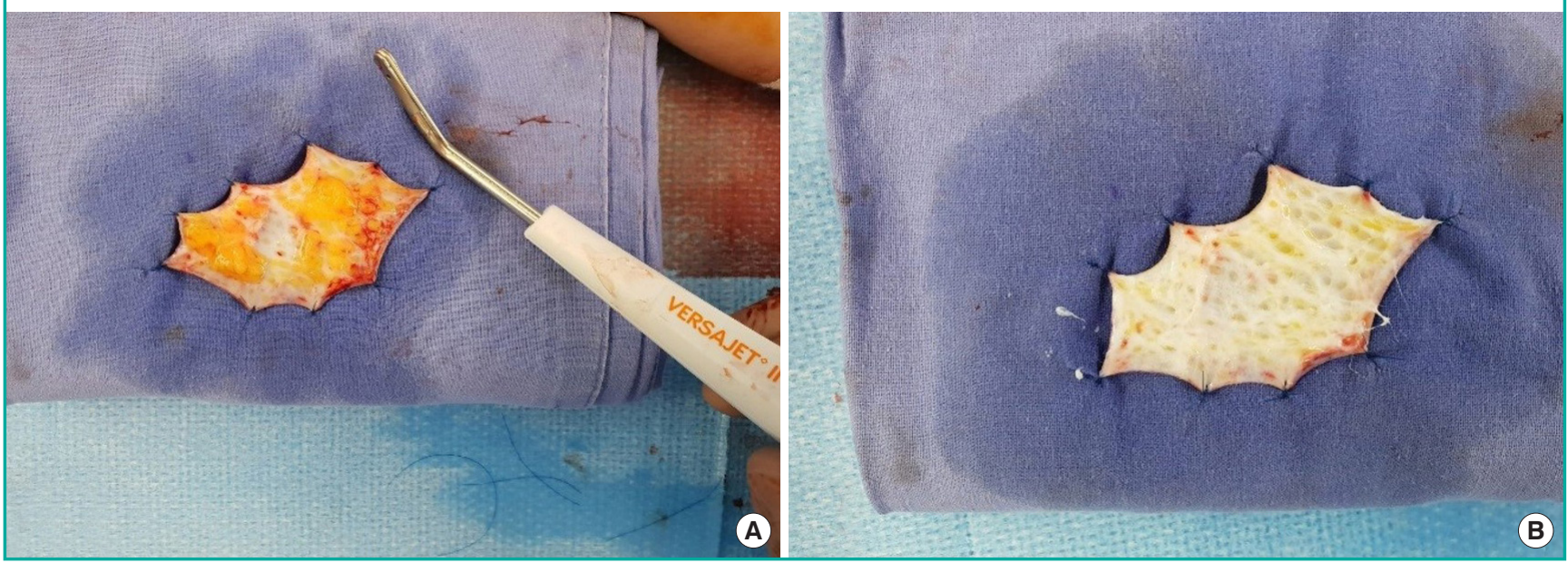
was obtained. As shown in Fig. 3, the graft was inset to the skin defect of the cubital fossa and sutured using nylon 5-0 sutures. A tie-over bolster was applied to keep the graft in place and was removed on postoperative day 7 . The grafted skin was shown to be healthy, and the graft was well-taken. During the outpatient follow-up period, the wound was observed to heal without any complications, such as infection, hematoma, or graft loss. The stitches were removed on postoperative day 14 . The viability

\section{Fig. 4. Postoperative 2 months (idea 1)}

By 2 months post-surgery, the graft was observed to have healed completely without scar contracture, and the range of motion of the elbow joint was preserved.

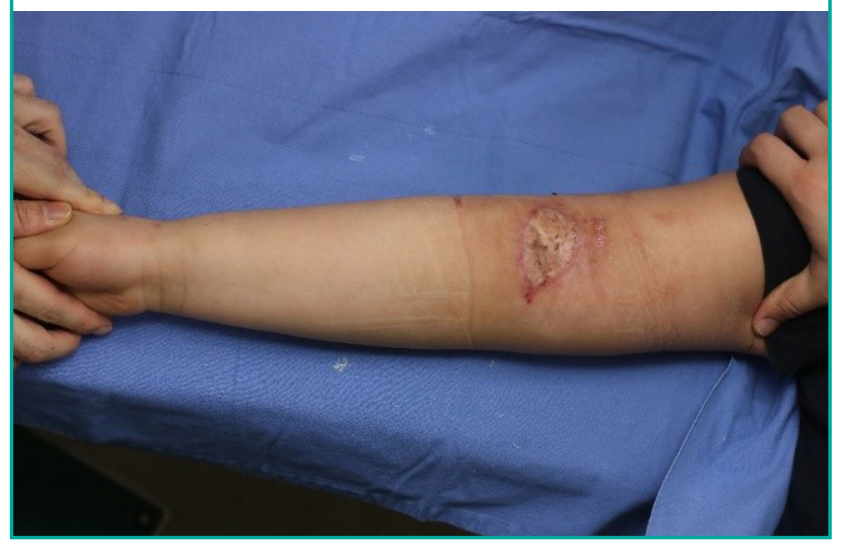

\section{Fig. 5. Recipient site preparation (idea 2)}

The size of the skin defect on the right proximal forearm area was observed to be $6 \mathrm{~cm} \times 4 \mathrm{~cm}$.

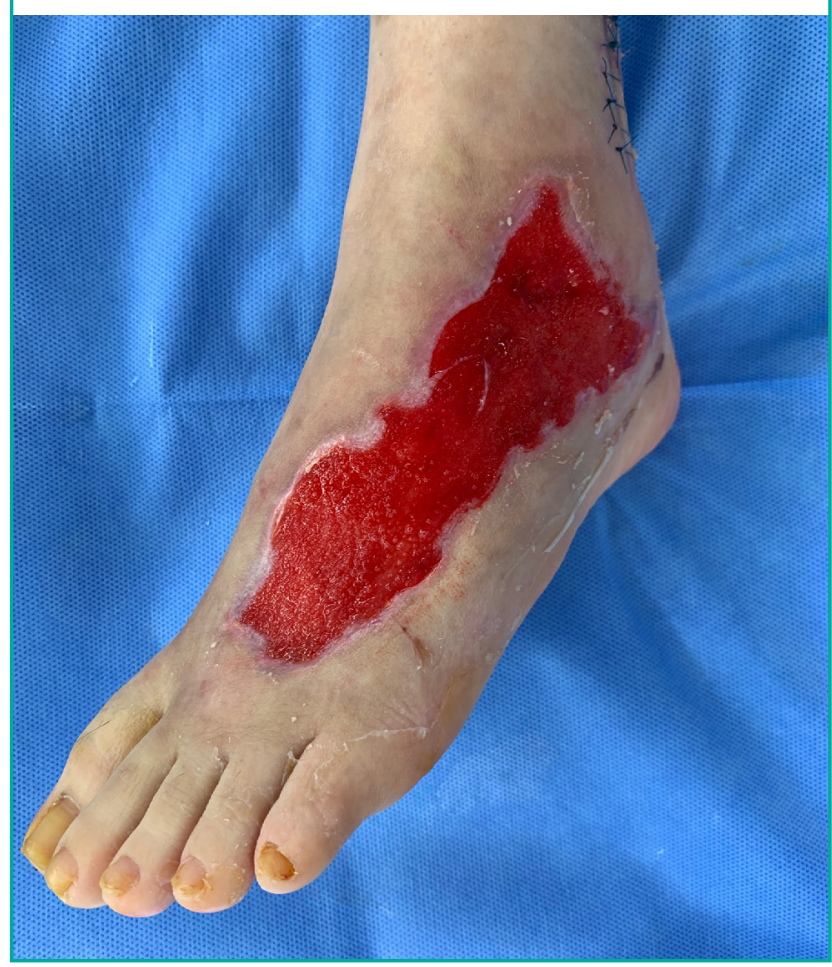

and successful healing of the graft was confirmed at a final follow-up appointment 2 months post-surgery (Fig. 4).

\section{Idea 2}

A 19-year-old woman was referred to the plastic surgery department due to a soft tissue defect of the dorsum of the left foot caused by cellulitis. The size of the defect was $12 \mathrm{~cm} \times 5 \mathrm{~cm}$ with a well-vascularized granulation bed (Fig. 5). The donor skin was harvested from the left lower abdomen. Preparation of the donor skin (Fig. 6) was performed in the same manner as in idea 1. Negative-pressure wound therapy was applied to the graft site instead of a tie-over dressing and was removed on postoperative day 4 . The grafted skin was healthy, and the graft was well-taken. The stitches were removed on postoperative day 14 . The viability and successful healing of the graft was confirmed at a final follow-up appointment 1 month post-surgery (Fig. 7).

\section{Fig. 6. Preparation of harvested donor skin (idea 2)}

The harvested skin was defatted and trimmed using the Versajet Hydrosurgery System. The desired thickness of the harvested skin was obtained.

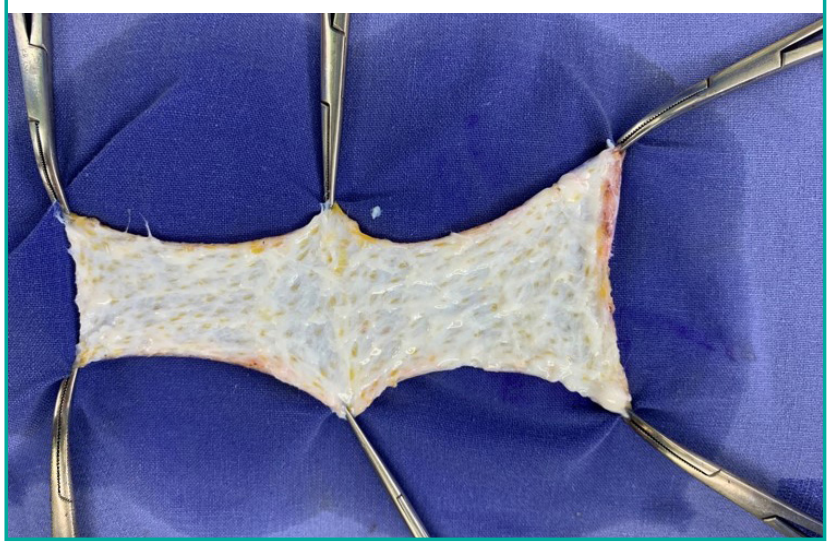

Fig. 7. Postoperative 1 month (idea 2)

By 1 month post-surgery, the graft was observed to have healed completely without any complications.

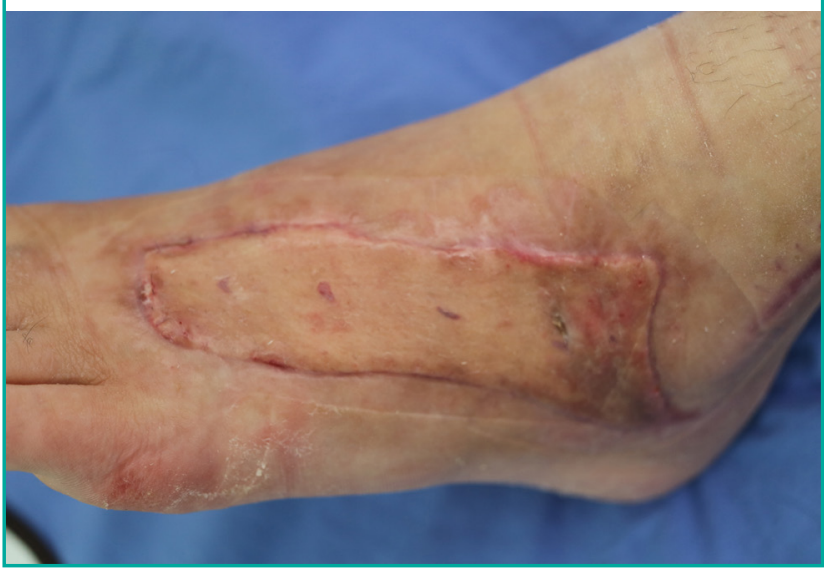




\section{DISCUSSION}

FTSG is recommended over STSG in areas where the range of motion of a joint is important. The grafted skin takes through plasmatic imbibition, capillary ingrowth, and revascularization from both the wound margins and the healthy wound beds [3]. Therefore, the thickness of the harvested donor skin is critical. It is very important to determine the thickness of the harvested skin in consideration of its effects on graft viability and possible postoperative contracture.

Once the donor skin is harvested, the thickness of the harvested skin is modified according to the surgeon's judgment. In traditional FTSG, surgical instruments such as scalpels, scissors, and Metzenbaum scissors have been manually used for this procedure. However, this method has several drawbacks: (1) it is a time-consuming procedure; (2) it is difficult to obtain an even thickness or a smooth surface of the harvested skin; and (3) the harvested skin can be unintentionally damaged during the resection of the undersurface. In spite of these drawbacks, there is a lack of publications suggesting other methods to regulate harvested skin thickness in FTSG. The Versajet Hydrosurgery System was developed in 1997 for soft tissue debridement of various types of wounds [4]. This apparatus is a hydrosurgical device that functions based on the principle of the Venturi effect. A saline solution is forced through a narrow window at a high velocity, creating a localized vacuum. The suction permits targeted tissue to be cut while aspirating debris from the site $[5,6]$.

Several studies have supported the efficacy and safety of the Versajet apparatus [2,5,7-10]. In addition, the authors of a separate study used this device to de-epithelialize the skin of the flap for coverage of pressure ulcer defects. That study reported that this procedure saved time and that the de-epithelialization results were uniform [11]. Similarly, the authors of the present study considered the possibility that the Versajet System could be applied to adjust the thickness of harvested skin in FTSG. The Versajet device is generally used to debride the wound bed of the recipient and then the handpiece is discarded. However, we surmised that it could be used for additional purposes.

In our first idea, as the patient had an intellectual disability, it was thought that flap surgery should be avoided due to the potential lack of postoperative cooperation by the patient. To preserve joint function, it was very important to obtain the appropriate thickness of the harvested skin in the FTSG procedure. Therefore, we decided to utilize the Versajet device in this case. Preparation of the harvested skin using hydrosurgery has the following advantages: (1) it requires less time than the method involving a scalpel; notably, in this case, it only took only $1 \mathrm{~min}$ ute to adjust the thickness of a segment of skin that measured 6 $\mathrm{cm} \times 4 \mathrm{~cm}$; (2) it enables the differentiation of tissue types, while saving viable tissue and accurately targeting and resecting unnecessary tissue by adjusting the power settings; (3) it can yield a smoother surface and a more uniform thickness throughout the entire undersurface than can be attained with manual debridement using a scalpel, and it also facilitates tight adhering of the graft to the wound bed, which may increase the chance of graft viability; and (4) surgeons can accurately and precisely control the mechanical power to adjust the thickness of the graft in consideration of the balance between postoperative contracture and the viability of the grafted skin.

In spite of these advantages, the high cost of this device can be a significant limitation of this method. In Korea, each handpiece costs about $\$ 400$. However, if Versajet use is already planned for debridement of the recipient site, it may be more cost-effective to also use this device for preparation of the harvested skin, rather than discarding the device after using it on the recipient site alone.

\section{NOTES}

\section{Conflict of interest}

No potential conflict of interest relevant to this article was reported.

\section{Ethical approval}

The study was approved by the Institutional Review Board of Kangbuk Samsung Hospital (IRB No. KBSMC 2019-07-052) and performed in accordance with the principles of the Declaration of Helsinki. Written informed consents were obtained.

\section{Patient consent}

The patients provided written informed consent for the publication and the use of their images.

\section{Author contribution}

Conceptualization: Shin HW. Data curation: Shin HW. Formal analysis: Choi SG. Methodology: Shin HW. Project administration: Yoon KC. Visualization: Choi SG. Writing - original draft: Choi SG. Writing - review \& editing: Shin HW. Approval of final manuscript: all authors.

\section{ORCID}

Seo Gil Choi https://orcid.org/0000-0002-8945-2278 Hyun Woo Shin https://orcid.org/0000-0003-4396-3395 Kun Chul Yoon https://orcid.org/0000-0001-9167-6716 


\section{REFERENCES}

1. Audrain H, Bray A, De Berker D. Full-thickness skin grafts for lower leg defects: an effective repair option. Dermatol Surg 2015;41:493-8.

2. Gravante G, Delogu D, Esposito G, et al. Versajet hydrosurgery versus classic escharectomy for burn débridment: a prospective randomized trial. J Burn Care Res 2007;28:720-4.

3. Pu LL, Coleman SR, Cui X, et al. Autologous fat grafts harvested and refined by the Coleman technique: a comparative study. Plast Reconstr Surg 2008;122:932-7.

4. Klein MB, Hunter S, Heimbach DM, et al. The Versajet water dissector: a new tool for tangential excision. J Burn Care Rehabil 2005;26:483-7.

5. Bekara F, Vitse J, Fluieraru S, et al. New techniques for wound management: a systematic review of their role in the management of chronic wounds. Arch Plast Surg 2018;45: 102-10.

6. Granick MS, Posnett J, Jacoby M, et al. Efficacy and cost-ef- fectiveness of a high-powered parallel waterjet for wound debridement. Wound Repair Regen 2006;14:394-7.

7. Bibbo C. VERSAJET hydrosurgery technique for the preparation of full thickness skin grafts and the creation of retrograde split thickness skin grafts. J Foot Ankle Surg 2010;49: 404-7.

8. Hong CC, Nather A, Lee JK, et al. Hydrosurgery is effective for debridement of diabetic foot wounds. Ann Acad Med Singapore 2014;43:395-9.

9. Kakagia DD, Karadimas EJ. The efficacy of Versajet $^{\mathrm{TM}}$ hydrosurgery system in burn surgery: a systematic review. J Burn Care Res 2018;39:188-200.

10. Legemate CM, Goei H, Gostelie OF, et al. Application of hydrosurgery for burn wound debridement: an 8-year cohort analysis. Burns 2019;45:88-96.

11. Oh SH, Song SH, Kyung H, et al. VersaJet Hydrosurgery System: an effective device for flap deepithelialization. J Craniofac Surg 2014;25:e390-2. 